Original scientific paper

\title{
DATA-DRIVEN ROBUST MODEL FOR CONTAINER SLOT ALLOCATION WITH UNCERTAIN DEMAND
}

\author{
Ma, X. Y.*; Lin, Y.*,"\# \& Ma, Q. W.** \\ * School of Economics and Management, Southeast University, Nanjing, 211189, China \\ ${ }^{* *}$ Information Center, China Tobacco Fujian Industrial Co., Ltd., Xiamen, 361012, China \\ E-Mail: linyu_fjtic@163.com $\left({ }^{\#}\right.$ Corresponding author $)$
}

\begin{abstract}
The global container transportation market is complex and changeable, resulting in high uncertainty of container cargo demand. Effective container slot allocation (CSA) decisions are difficult for shipping companies to make. A data-driven robust model was developed for optimizing the CSA under highly uncertain cargo demands to maximize the revenues of shipping companies. The features of the empty container transportation were integrated into the model due to the imbalance of container import and export. The Copula method was employed to construct the uncertain set to deal with the limited historical demand information. Moreover, the model can be transformed into a simple and manageable robust optimization problem by introducing the protection level. Finally, the effectiveness of the optimal robust CSA policy was verified by numerical examples. Results demonstrated that the robust optimization model effectively balances the relationship between the revenue and the risk preference of shipping companies and maximizes the revenue by using limited cargo demand information. The optimal robust slot allocation policy is more stable under the heavy tail cargo demand.

(Received in August 2021, accepted in November 2021. This paper was with the authors 1 month for 1 revision.)
\end{abstract}

Key Words: Copula Method, Data-Driven, Robust Optimization, Container Slot Allocation

\section{INTRODUCTION}

The world trades volume remains rapid growth with the prosperity of the world's economy. Several advantages, such as ensuring cargo safety, saving packaging materials, and simplifying operating procedures have promoted the rapid development of container transportation. The data show that 6153 container ships were in operation worldwide in September 2020, with a total capacity of 24,062,901 TEU [1]. Shipping companies can improve the efficiency of container deliveries through container slot allocation (CSA) decisions [2].

The cargo demand is one of the essential considerations when making CSA decisions. The cargo demand is often assumed to be deterministic or stochastic with known probability distribution [3-5]. However, the cargo demand became highly uncertain due to the severe international situation, changeable natural environment, fluctuating market conditions, free cancellation of customer's booking slot [6]. For example, the global supply chain was seriously hit by the impact of COVID-19 in 2020. Strict export restrictions at the early stage led to a decline in container slot. After the COVID-19 era, the sudden rise in the demand for epidemic prevention material resulted in an increase in container slot. These uncertainties intensify the fluctuation of cargo demands. The cargo demand cannot be described with the known probability distribution based on historical data as before, resulting in the difficulty of the shipping companies to make reasonable CSA decisions [7, 8]. In addition, shipping companies have different risk preferences. Therefore, shipping companies must find new methods for dealing with uncertain demand and making CSA decisions in terms of their risk preference in the complex market.

Empty container transportation is also a big problem. The imbalance of trade in the world has caused the disparity of container quantity among ports for a long time. In the fierce market environment, the freight is difficult to improve, and the empty container transportation costs 
are high [9]. The situation of "One container is hard to get" during the COVID-19 further exacerbates the seriousness effects of this problem. Accordingly, the Thai Port Authority issued a policy of subsidy of 1000 strains per empty container for the shortage of containers for exporters. In the CSA decisions, the empty container allocation should be considered to maintain the long-term balance of container quantity among ports.

This work studies the CSA problem considering empty container transportation under the highly uncertain cargo demands among ports. Given that the shipping company only obtains the limited historical demand data, a data-driven method is proposed to analyse the correlation of demand, and the corresponding uncertain set is constructed. The robust optimization model of CSA is developed to obtain the optimal robust slot allocation policy based on the protection level. The main contribution in this study can be summarized as follows: the robust optimization model can construct the uncertain set of the cargo demand based on the limited historical demand information, which effectively copes with the difficulty caused by the unavailable probability distribution under the highly uncertain cargo demand. In addition, the risk preference is integrated into the robust optimization model. Accordingly, the relationship between the revenue and the risk preference can be balanced in the complex market.

The rest of this study is arranged as follows. Section 2 provides a literature review. The problem description, notation, and basic assumptions are introduced in Section 3. The mathematical model of CSA is proposed in Section 4, and the data-driven robust optimization method is introduced. The numerical analysis is reported in Section 5. Finally, the conclusion and future research directions are presented in Section 6.

\section{STATE OF THE ART}

In the past, several researchers focused on the CSA problem. Feng and Chang [3] studied the CSA for a specific route with the consideration of ship capacity, container demand, and empty container supply. Zurheide and Fischer $[4,10]$ applied revenue management to solve the CSA problem considering market segmentation, route transhipment, and bidding price. Hu et al. [11] analysed the characteristics of contract customers and scattered customers, and proposed a maximum profit model with empty container transportation. In the above literature, the container cargo demands are assumed to be known and deterministic. It is more realistic to describe the demands using stochastic variables in a complex and changeable container transportation market.

Some studies considered the uncertainty of cargo demand. Lu et al. [2] established the CSA model to meet the seasonal demand of linear shipping for maximizing the potential profit for each round trip. Fu et al. [12] applied a robust optimization method to solve the CSA with minimum quantity commitment under uncertainty demand. Ting and Tzeng [13] proposed a dual objective CSA model considering carrier freight and customer satisfaction. Wang et al. [14] proposed a static oversold side strategy and a delayed delivery strategy to deal with the deviation between the predicted and the actual demand. Huang [9] solved the problem of CSA with empty containers under bounded uncertain demand and added container volume and weight into the constraints. Zhao et al. [15] established a robust optimization model for CSA by using basic descriptive statistics, such as mean, upper/lower bound, and variance. Wang and Meng [7] proposed three different prediction models for the number of containers in the intercontinental long-distance transportation service: piecewise linear regression, autoregression, and artificial neural network. Zhao et al. [6] proposed a data-driven two-stage stochastic nonlinear nonconvex programming model for the cancellation analysis of an intercontinental container shipping slot. In the aforementioned literature, the cargo demand can be accurately described by the known probability distributions. In practice, accurately estimating the probability distribution of a cargo demand in a short period based on the limited 
historical demand data is difficult. In addition, the effects of the shipping company's risk preferences in the complex and uncertain market are not discussed.

Robust optimization (RO) was first proposed by Soyster [16], and it has been widely studied and greatly expanded since then. Several main forms are in the process of development, such as the RO model based on the scenario set [17], the improved RO model [18], and the RO model with the protection level [19]. Under RO, the sub-optimal solution is still feasible and close to the optimal solution. This feature also makes RO widely used in various fields, such as jobshop scheduling [20], dynamic transportation [21], service network [22], reverse logistics [23], and disaster relief [24]. In recent years, RO is gradually combined with data-driven technology to better deal with uncertainty. Ning and You [25] used machine learning and big data analysis technology to integrate RO for data-driven decision-making. Zhang et al. [26] proposed a method based on chance constraints and robust constraints to solve the optimization problem of matrix uncertainty for continuous uncertain constraints. In the literature discussed above, the uncertain sets in the RO models have been assumed to be known or constructed based on the sufficient data. However, the shipping company in this study is assumed to only obtain the limited historical demand data.

In summary, this study constructs the uncertain set by using the limited historical demand data based on a Copula method and develops a robust optimization model of CSA with the protection level. The shipping company can balance the relationship between revenue and model robustness through the setting of the protection level and then provides robust slot allocation policies in the different risk preferences.

\section{METHODOLOGY}

\subsection{Problem description and assumption}

A container shipping company provides a series of cargo services to multiple container shipping lines. The shipping lines consist of $p$ flight segments, $p+1$ affiliated ports, and $q$ port pairs, as shown in Fig. 1. The shipping company carries $K$ different types of cargoes (i.e., refrigerated cargoes and ordinary cargoes), and the revenue incurred by offering each type of cargo service is different. In addition, there are a large number of empty containers in some ports due to the trade imbalance, which incurs high costs to transport empty containers. The shipping company should allocate a certain number of container slot and carry a specific quantity of empty containers to balance empty containers among ports.

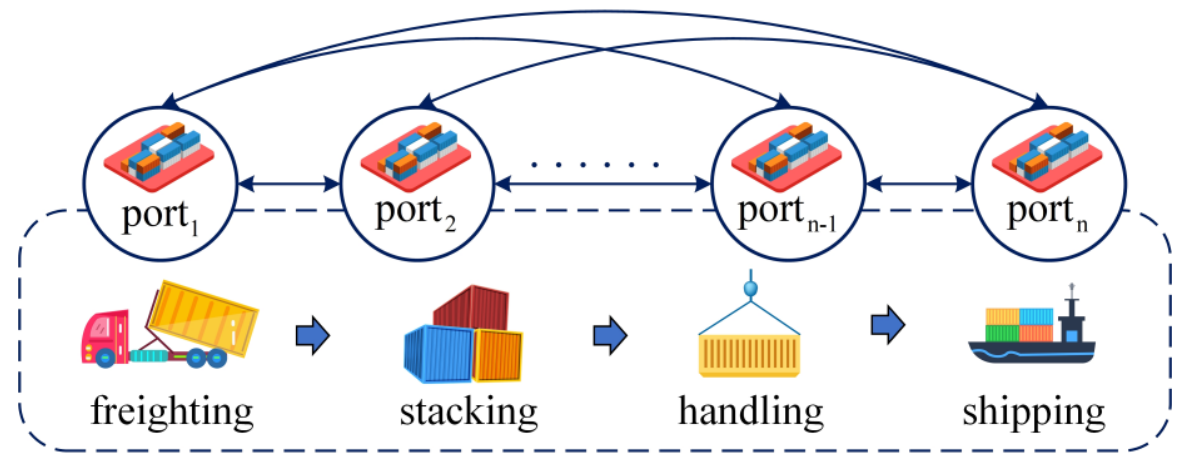

Figure 1: Routes and ports diagram.

The shipping market is uncertain and changeable due to the complex international trade situation. The shipping company cannot easily match the stochastic demand with limited capacity for the ports. The shipping company only obtains the historical cargo demand data over a short period. Given the limitation in historical data, a data-driven robust optimization 
model is formulated in this study to obtain the robust CSA policy. The other key assumptions of the model are summarized as follows:

(1) The containers, including heavy and empty containers, are assumed to be all 20 -foot standard containers. The other types of containers are not to be considered.

(2) The total weight of all types of cargoes (including the self-weight of the containers) carried on the ship does not exceed the deadweight of the ship.

(3) The speed of the ship is the same on the voyage and between ports. The average fuel consumption, terminal handling charges, management costs, and other factors are the same, and the incurred differences are not considered in the model.

The notation used in the model is shown in Table I.

Table I: Description of the notation.

\begin{tabular}{|c|l|}
\hline Parameter & \multicolumn{1}{|c|}{ Definitions } \\
\hline$p$ & Number of flight segments \\
\hline$q$ & Number of port pairs \\
\hline$\Omega$ & Set of port pairs where the shipping company stops, $\Omega=\{(i j)\}$ \\
\hline$\Omega_{s}$ & Set of starting ports \\
\hline$\Omega_{d}$ & Set of destination ports \\
\hline$k$ & Types of cargoes carried by shipping companies $(k=1,2, \ldots, K)$ \\
\hline$C_{m}$ & Maximum number of the available container slot on flight segment $m$ \\
\hline$r_{i j}^{k}$ & Unit revenue of the cargoes of category $k$ from port $i$ to port $j$ \\
\hline$d_{i j}^{k}$ & Demand of the cargoes of category $k$ from port $i$ to port $j$, random variables \\
\hline$u_{i j}^{k}$ & Minimum shipment volume of the cargoes of category $k$ from port $i$ to port $j$ \\
\hline$c_{i j}$ & Unit cost of carrying empty containers from port $i$ to port $j$ \\
\hline$A_{i j m}^{k}$ & $\begin{array}{l}\text { 0-1 variable, when carrying the cargoes of category } k \text { between }(i j) \text { through flight } \\
\text { segment } m, A_{i j m}^{k}=1 ; \text { otherwise, } A_{i j m}^{k}=0\end{array}$ \\
\hline \multicolumn{2}{|l}{} \\
\hline$x_{i j}^{k}$ & Number of container slot allocated from $i$ to $j$ for cargoes of category $k$ \\
\hline$y_{i j}$ & Number of empty containers transported from $i$ to $j$ \\
\hline
\end{tabular}

\subsection{The model}

The CSA model is formulated as follows:

$$
\begin{gathered}
\max z=\sum_{(i j) \in \Omega} \sum_{k=1}^{K} r_{i j}^{k} x_{i j}^{k}-\sum_{(i j) \in \Omega} c_{i j} y_{i j} \\
\text { s.t. } \sum_{(i j) \in \Omega}\left(\sum_{k=1}^{K} A_{i j m}^{k} x_{i j}^{k}+y_{i j}\right) \leq C_{m}, m=1,2, \ldots, M \\
x_{i j}^{k} \leq d_{i j}^{k} \\
x_{i j}^{k} \geq u_{i j}^{k} \\
\sum_{i:(i j) \in \Omega} \sum_{k=1}^{K} x_{i j}^{k}+\sum_{i:(i j) \in \Omega} y_{i j}-\sum_{i:(j i) \in \Omega} \sum_{k=1}^{K} x_{i j}^{k}-\sum_{i:(j i) \in \Omega} y_{i j}=0, \forall j \in \Omega_{d} \\
x_{i j}^{k}, y_{i j} \in N \cup\{0\}
\end{gathered}
$$

The objective function Eq. (1) maximums the total revenue, including the revenue from transporting heavy containers and the cost of transporting empty containers. Eq. (2) limits the number of containers transported between ports to no more than the maximum transport 
capacities. Eq. (3) limits the number of containers allocated to no greater than the demands. Eq. (4) guarantees that the number of containers allocated to the class of cargoes is not less than the minimum departure volume of this type of cargoes. Eq. (5) allows the number of containers unloaded and dispatched to be the same to maintain the balance of container quantity in each port. Finally, Eq. (6) specifies that the variables are nonnegative integers.

\subsection{Data-driven robust optimization method}

Since the demand variables $d_{i j}^{k}$ in Eq. (3) are stochastic, the model cannot be directly solved. Next, we introduce a data-driven robust optimization method to deal with $d_{i j}^{k}$.

A direct transformation method to deal with uncertain demand is to directly replace $d_{i j}^{k}$ by the expectation, which is simple and efficient. In practice, the demand fluctuations are often complex due to the changeable natural environment and trade policies. A large deviation can be observed between the results obtained from the nominal value and the actual one. Furthermore, the deviation may not be accurately described since the shipping companies only master limited historical demand data. The expectation method cannot provide a better decision-making basis for the shipping companies. In view of the above situation, the datadriven robust optimization method can fully utilize the historical data to deal with the uncertain demand. The transformation steps are as follows:

Step 1: Uncertain correlation analysis

The probability density functions (PDFs) are fitted by kernel density based on the limited historical data from the shipping companies, and the correlation coefficients are calculated. The kernel function with the Gaussian kernel function is used to estimate PDFs [25].

The main steps of estimating the joint PDF based on Copula method are as follows: (1) selecting the appropriate Copula and estimating the multivariate cumulative distribution function (CDF); (2) generating relevant uncertain scenarios to supplement limited historical data; (3) hypothesis testing for estimating CDF; and (4) deciding whether to accept the estimated results. The detailed steps of the Copula method refer to [25].

Step 2: Uncertain set construction

The relevant uncertain scenarios generated by the joint PDF, which are combined with historical data (original data) to form a comprehensive data set. On this basis, the method in Zhang et al. [27] is used to transform the uncertain cargo demand into the following form:

$$
d_{i j}^{k}=\alpha_{i j}^{k, 1-\gamma_{i}}+\xi_{i j}^{k} \omega_{i j}^{k, 1-\gamma_{i}}
$$

In Eq. (7), $\alpha_{i j}^{k, 1-\gamma_{i}}=\frac{F_{d_{i j}^{k}}^{-1}\left(\gamma_{i} / 2\right)+F_{d_{i j}^{k}}^{-1}\left(1-\gamma_{i} / 2\right)}{2}$ and $\omega_{i j}^{k, 1-\gamma_{i}}=\frac{\left|-F_{d_{i j}^{k}}^{-1}\left(\gamma_{i} / 2\right)+F_{d_{i j}^{k}}^{-1}\left(1-\gamma_{i} / 2\right)\right|}{2}$.

Here, $\alpha_{i j}^{k, 1-\gamma_{i}}$ represents the nominal value of the random demand variable, and $\omega_{i j}^{k, 1-\gamma_{i}}$ denotes the maximum fluctuation value of random demand variable. $F_{d_{i j}^{k}}^{-1}(\cdot)$ is the inverse estimated CDF. $\xi_{i j}^{k} \in[-1,1]$ is an interval variable used to control the range of uncertain variable, and $1-\gamma_{i}$ is the confidence level of the $i^{\text {th }}$ constraint. Therefore, the bounded form of continuous uncertain variables $d_{i j}^{k}$ can be expressed as follows:

$$
d_{i j}^{k} \in\left[\alpha_{i j}^{k, 1-\gamma_{i}}-\omega_{i j}^{k, 1-\gamma_{i}}, \alpha_{i j}^{k, 1-\gamma_{i}}+\omega_{i j}^{k, 1-\gamma_{i}}\right]
$$

Step 3: Robust optimization model

After the uncertain set is obtained, the basic model is transformed by the robust optimization method proposed by Bertsimas and Sim [19]. In this method, the protection level $\Gamma$ is set, which allows multiple variables in each constraint to change at the same time. That is, when the protection level is $\Gamma, \Gamma$ uncertain variables in the model are allowed to simultaneously change. When more than $\Gamma$ uncertain variables simultaneously change, there are still situations wherein 
the constraint conditions are violated. Accordingly, the transformation of Kim et al. [23] is used to deal with this situation. The total number of constraints with uncertain variables is regarded as the total uncertainty, and the protection level is evenly distributed to each constraint. The constraint condition of $d_{i j}^{k}$ in the basic model can be rewritten as follows:

$$
x_{i j}^{k} \leq\left(\alpha_{i j}^{k, 1-\gamma_{i}}-\frac{\Gamma}{|J|} \omega_{i j}^{k, 1-\gamma_{i}}\right)
$$

The solvable data-driven robust optimization model after the above transformation steps is composed of Eqs. (1) to (2), Eqs. (4) to (6), and Eq. (9).

\subsection{Model robustness}

According to [19], the probability that the $i^{\text {th }}$ constraint is not violated satisfies the following:

$$
\operatorname{Pr}\left(x_{i j}^{k} \leq d_{i j}^{k}\right) \geq 1-B(n, \Gamma)
$$

where

$$
B(n, \Gamma)=\frac{1}{2^{n}}\left\{(1-\mu) \sum_{l=\lfloor v\rfloor}^{n}\left(\begin{array}{l}
n \\
l
\end{array}\right)+\mu \sum_{l=\lfloor v\rfloor+1}^{n}\left(\begin{array}{l}
n \\
l
\end{array}\right)\right\}=\frac{1}{2^{n}}\left\{(1-\mu)\left(\begin{array}{c}
n \\
\lfloor v\rfloor
\end{array}\right)+\sum_{l=\lfloor v\rfloor+1}^{n}\left(\begin{array}{l}
n \\
l
\end{array}\right)\right\}
$$

In Eq. (11), $n$ represents the number of uncertain variables, $v=\frac{\Gamma+n}{2}, \mu=v-\lfloor v\rfloor$.

The robustness of the model can be enhanced by adjusting the protection level; hence, decision-makers can combine their own risk preferences to balance the robustness of the model and the cost of achieving robustness.

\section{RESULT ANALYSIS AND DISCUSSION}

\subsection{Example description}

The performance of the proposed model and transformation method is verified through numerical experiments. Numerical experiments were conducted using MATLAB R2017b software on an Intel ${ }^{\circledR}$ Core $^{\mathrm{TM}}$ i5-7300 $2.5 \mathrm{GHz}$ CPU computer with $4 \mathrm{~GB}$ of RAM. In this section, a shipping company with two routes, three affiliated ports, and a total of six-port pairs is considered. The shipping company transports refrigerated and ordinary cargoes in a certain period. No correlation exists between the demands of the two types of cargoes. The maximum number of container slot available for the shipping company on each route is 1500 TEU.

The unit revenue obtained by carrying the same cargoes and the unit cost of empty containers between port pairs are not exactly same due to the distance between port pairs, road conditions, and other factors. The demand quantity of cargoes is also different. The values of these parameters in this study are shown in Table II and Table III.

In this study, we compare the real distribution with normal distribution and lognormal distribution and generate historical demand sample data based on the two distributions. Then, the data-driven robust optimization method is used according to the historical data.

Table II: Model related parameter values.

\begin{tabular}{|c|c|c|c|c|}
\hline Parameters & $p$ & $q$ & $K$ & $C_{m}$ \\
\hline Value & 2 & 6 & 2 & 1500 \\
\hline
\end{tabular}

The relevant parameters of normal distribution used to generate historical demand sample data are randomly generated in the interval shown in Table IV. 
Table III: Model related parameter values.

\begin{tabular}{|l|c|c|c|c|c|c|}
\hline \multicolumn{1}{|c|}{ Port pair $(i \rightarrow j)$} & $\mathbf{1 \rightarrow \mathbf { 2 }}$ & $\mathbf{1} \rightarrow \mathbf{3}$ & $\mathbf{2 \rightarrow \mathbf { 1 }}$ & $\mathbf{2 \rightarrow \mathbf { 3 }}$ & $\mathbf{3 \rightarrow \mathbf { 1 }}$ & $\mathbf{3 \rightarrow \mathbf { 2 }}$ \\
\hline Unit revenue of refrigerated cargo $\left(r^{1}{ }_{i j}\right)$ & 280 & 210 & 240 & 230 & 200 & 200 \\
\hline Unit revenue of ordinary cargo $\left(r^{2}{ }_{i j}\right)$ & 140 & 170 & 150 & 120 & 120 & 170 \\
\hline Unit cost of empty container $\left(c_{i j}\right)$ & 20 & 11 & 22 & 16 & 20 & 20 \\
\hline Minimum shipment volume of refrigerated cargo $\left(u^{1}{ }_{i j}\right)$ & 45 & 35 & 34 & 26 & 24 & 29 \\
\hline Minimum shipment volume of ordinary cargo $\left(u^{2} i j\right)$ & 40 & 36 & 35 & 26 & 25 & 20 \\
\hline
\end{tabular}

The parameters of lognormal distribution can be obtained by the following formula:

$$
\mu=\ln (E(X))-\frac{1}{2} \ln \left(1+\frac{\operatorname{var}(X)}{E(X)^{2}}\right), \sigma^{2}=\ln \left(1+\frac{\operatorname{var}(X)}{E(X)^{2}}\right)
$$

$E(X)$ and $\operatorname{var}(X)$ are the mean and variance of the real normal distribution, respectively.

Table IV: Generation interval of cargo demand-related parameters.

\begin{tabular}{|c|c|c|}
\hline Parameter & $\mu_{i j}^{k}$ & $\sigma_{i j}^{k}$ \\
\hline Interval & {$[600,800]$} & {$[200,300]$} \\
\hline
\end{tabular}

\subsection{Results analysis}

Twelve groups of data with two different distributions are randomly generated in the interval. Taking $d_{12}^{1}$ and $d_{21}^{1}$ as an example, the PDFs of two demands are listed in Fig. 2.
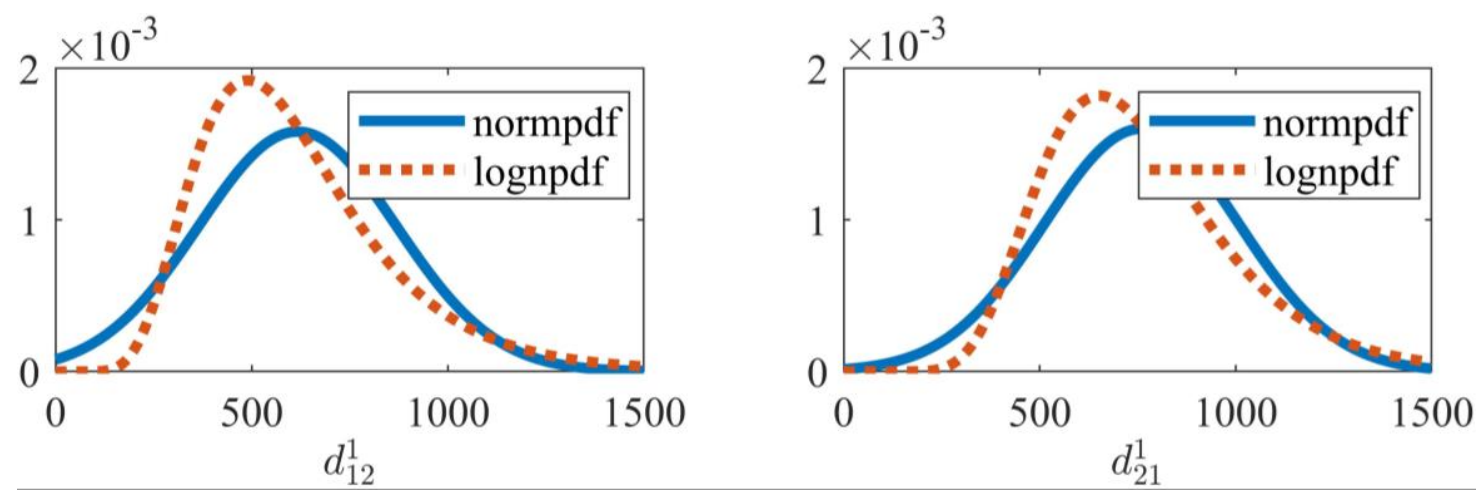

Figure 2: PDFs of two different distributions among port pairs.

From Fig. 2, the PDF of lognormal distribution moves to the right, and it is more concentrated. Next, the Kendall correlation coefficients $(K C C)$ of the demands are calculated.

When the absolute value of $K C C$ is greater than 0.1 , the demands between the two ports are related. Based on Copula theory, the Gaussian Copula function in the elliptic Copula function cluster is selected to obtain the appropriate joint PDF. Furthermore, the Monte Carlo method is used to generate a certain amount of simulated data, resulting in a comprehensive data set with the original data to supplement the uncertain scenario. 

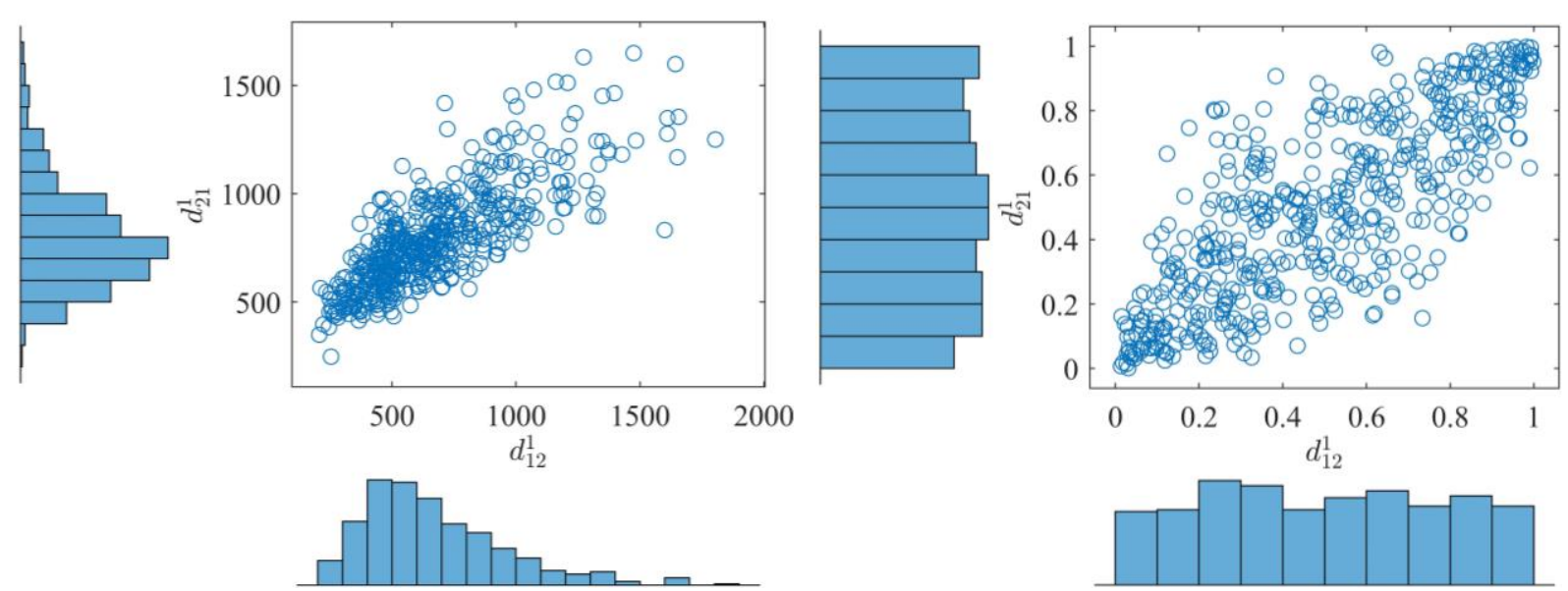

Figure 3: Correlation image of $d_{12}^{1}$ and $d_{21}^{1}$ (500 simulated dependent values in the left and 500 simulated dependent values transformed to $U(0,1)$ in the right).

From Fig. 3, the Kendall correlation of $d_{12}^{1}$ and $d_{21}^{1}$ is 0.4577 . The two factors have a high correlation. The estimated joint PDF is used to generate 500 simulated data, which are combined with the original data to form a comprehensive data set. The kernel density is used to fit the distribution characteristics of the data. The nominal value and the maximum fluctuation value of the demand random variables are calculated to obtain the uncertain set. In this example, let $\gamma_{i}=0.1$ to obtain $\alpha_{12}^{1,0.9}=665, \omega_{12}^{1,0.9}=361, \alpha_{21}^{1,0.9}=720$, and $\omega_{21}^{1,0.9}=322$. Therefore, the bounded set form of the two is as follows: $d_{12}^{1} \in[304,1026], d_{21}^{1} \in[398,1042]$.

In both cases, let $\gamma_{i}=0.1$. The model results under real distribution and fitting distribution can be obtained. The CSA schemes of real normal distribution and fitting normal when $\Gamma=12$ are shown in Figs. 4 and 5. Other situations are similar and will not be listed here.

From Figs. 4 and 5, we find that the robust solutions obtained by the data-driven method are closed to the optimal solutions with known distributions. It implies that the effectiveness of the robust optimization method in dealing with uncertain demands.

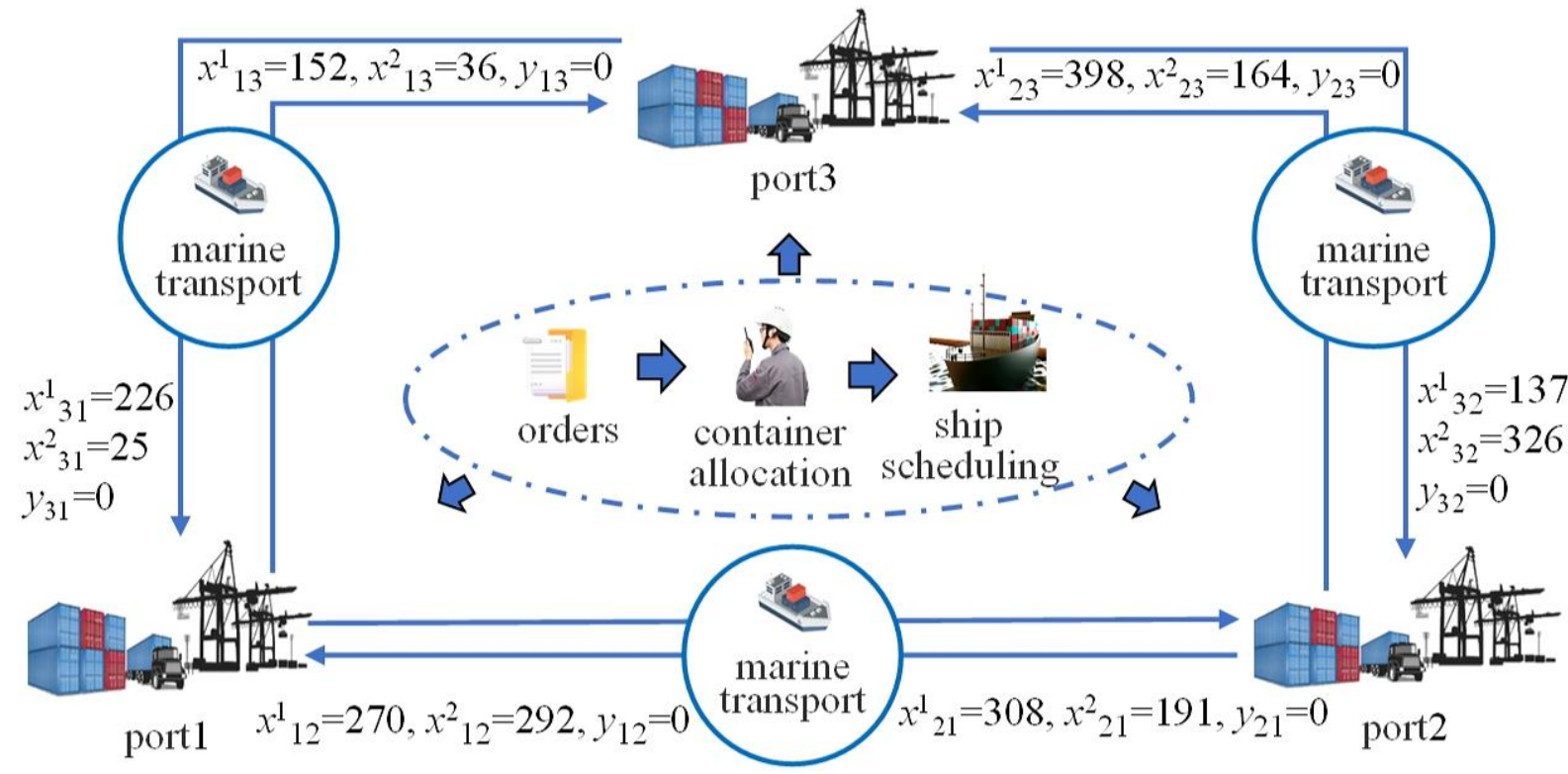

Figure 4: CSA scheme under real normal distribution when $\Gamma=12$. 


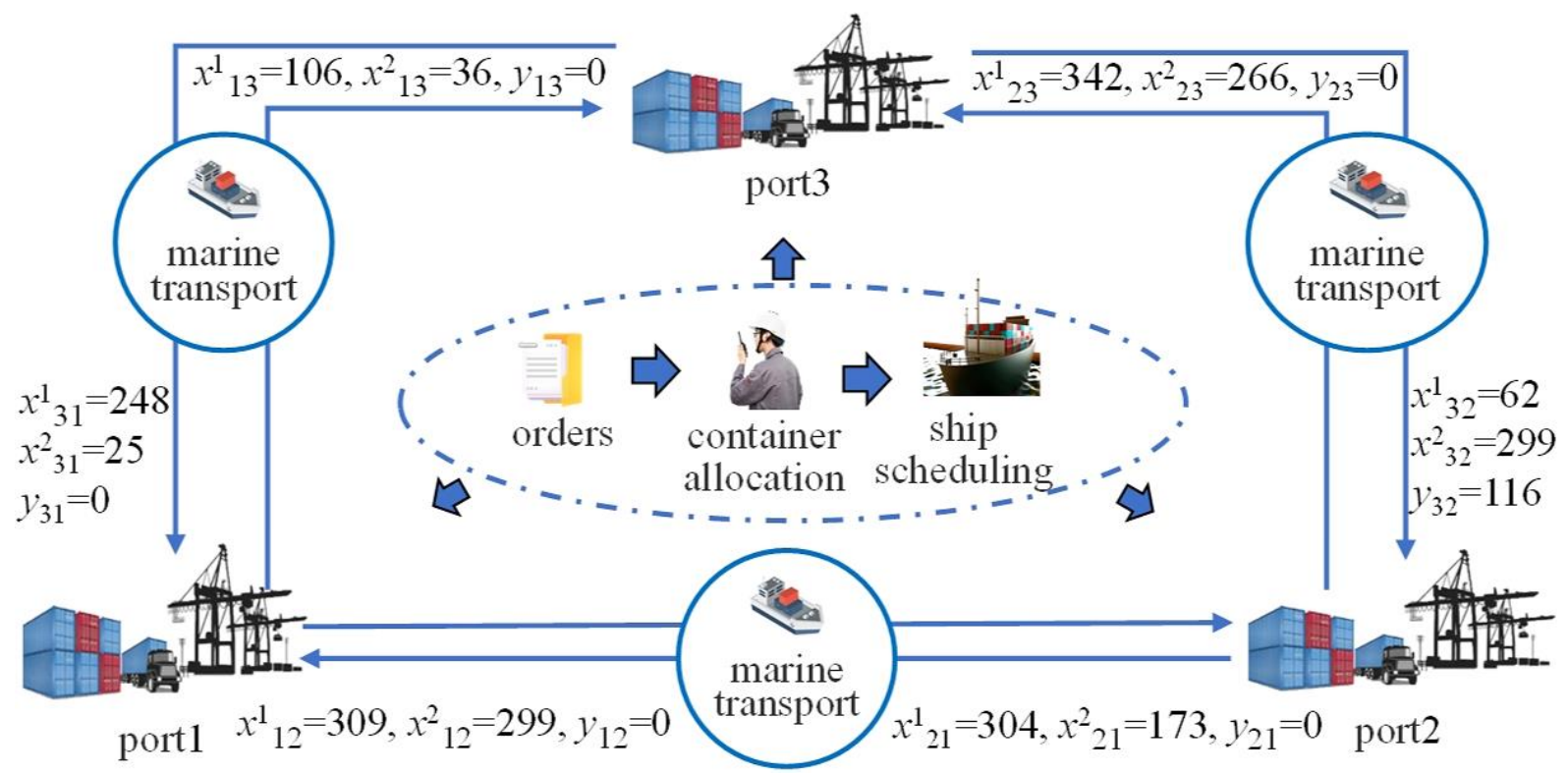

Figure 5: CSA scheme under fitting normal distribution when $\Gamma=12$.

\subsection{Effects of protection level and confidence level}

The model solving process was looped 500 times to eliminate the randomness in the simulation process of the example, and the average of the effective values of the 500 results is taken as the final result. When $\gamma_{i}=0.1$, the robustness of the model and the change of revenue with $\Gamma$ under the two distributions are shown in Fig. 6.

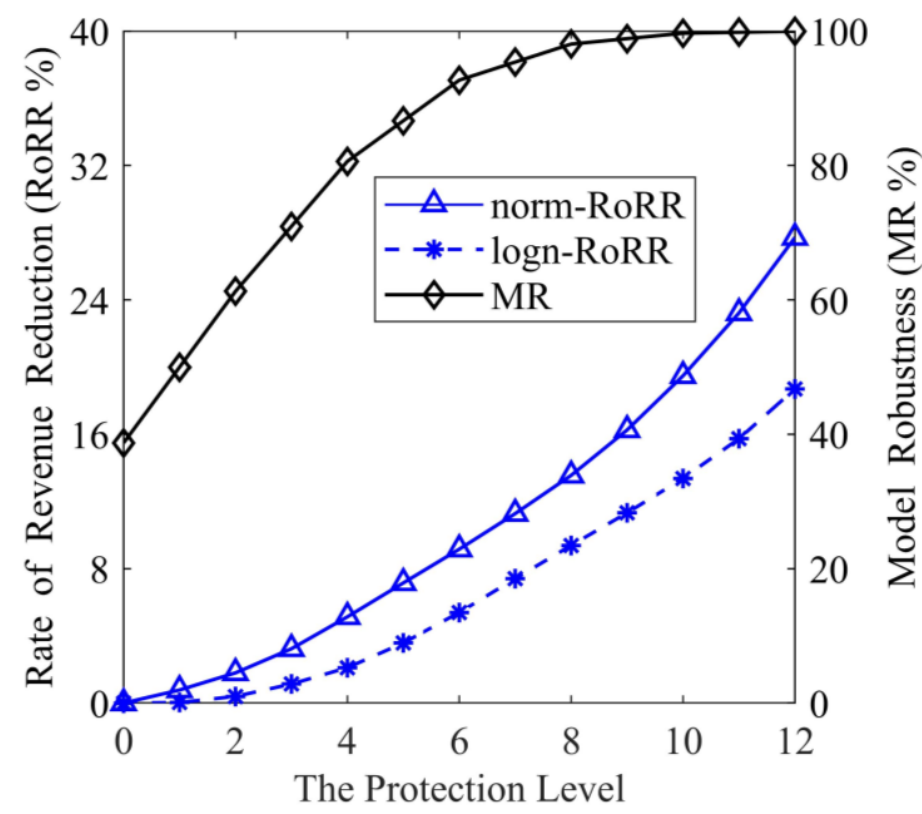

Figure 6: Robustness and revenue variation of the model under two distributions.

Under the two distributions, the robustness of the model increases with the increase in the protection level. The more demand uncertainties are considered, which leads to the reduction in revenue. For example, when $\Gamma=12,12$ random variables can change. The robustness of the model is close to $100 \%$, and the probability of constraint violation in the model becomes extremely small; however, the according revenue is the lowest. The strong robustness of the model leads to the over-conservatism. When $\Gamma=6$, the model has more than $90 \%$ robustness, 
and the revenue is only decreased by approximately $5 \%$ to $8 \%$. Hence, the decision-makers can obtain high model robustness with less cost, which is a more reasonable scheme.

The total revenue reduction rates are compared in two cases with between the normal distribution and the lognormal distribution. When $\gamma_{i}=0.1$, the total revenue reduction rate with the lognormal distribution is approximately $7 \%$, lower than that with the normal distribution. The reason is that the lognormal distribution is more concentrated. Although there is a large space for upward fluctuation, the confidence interval established in solving the model excludes some extreme cases with small probability. Accordingly, the fluctuation interval of the demand random variables following lognormal distribution is smaller and more stable than that conforming to normal distribution to a certain extent.

Under different confidence levels $\gamma_{i}=(0.05,0.1,0.2)$, the decrease in total revenue caused by the change of protection level is also different. Fig. 7 shows the change of the total revenue of two distributions with $\Gamma$ under three confidence levels.

The total revenue reduction rates under three confidence levels gradually increase with the increase in the protection level, indicating that the decision-maker needs to pay the cost in exchange for the robustness of the model. When the value of $\gamma_{i}$ increases, that is, the confidence level decreases, the influence of the robustness on the decrease of the total revenue decreases. This phenomenon occurs because the confidence interval of uncertain demand variables excludes many values with a low-probability of occurrence under the low confidence level, and the demand volatility is less considered. In each confidence level, the slope of the yield reduction curve gradually increases, indicating that the total revenue rapidly decreases when $\Gamma$ increases at the end, and the decision-maker needs to pay expensive costs to achieve the strongest robustness. Therefore, decision-makers should choose the appropriate scheme according to their needs and risk preference to balance the robustness of the model and the cost of achieving robustness.

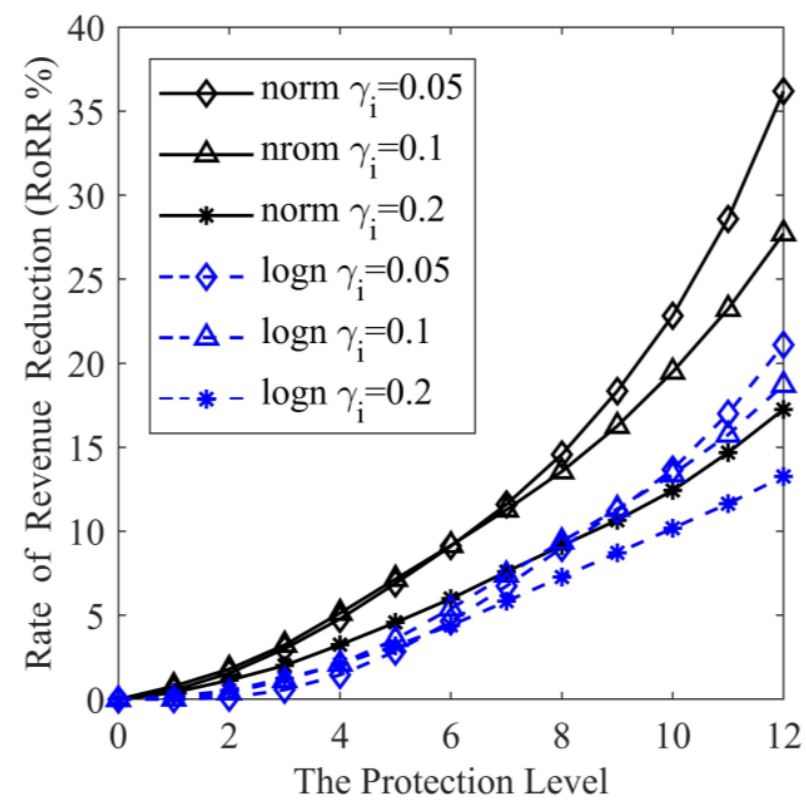

Figure 7: Total yield reduction rate of the model under two distributions; $\gamma_{i}=(0.05,0.1,0.2)$.

\section{CONCLUSION}

The traditional probability distribution cannot be accurately described in the highly uncertain cargo demand. It is difficult for shipping companies to make reasonable slot allocation decisions. Under the highly uncertain cargo demand, the data-driven robust optimization model for CSA for maximizing the revenue of companies was established. Based on the short-term limited 
historical demand data, the Kendall correlation coefficient was used to analyse the correlation of cargo demand among ports, and the Copula-based method was introduced to estimate the joint probability density function. Then, the uncertain sets are constructed. On this basis, the protection level was incorporated considering the risk preference of container shipping companies, and the model was transformed into a simple and manageable robust optimization problem. The effectiveness of the robust optimization model for CSA was verified with numerical simulations. The following conclusions could be drawn:

(1) Based on the short-term limited historical demand data, the CSA problem can be effectively solved by the data-driven robust model under a highly uncertain cargo demand.

(2) The model effectively balances the relationship between revenue and robustness and achieves the goal of maximizing the revenue of companies under different risk preferences. The risk preference is more conservative with the increase in the protection level, the decisionmaking emphasizes more robustness, and the revenue will be lower. Shipping companies can choose a reasonable CSA scheme according to their own risk preference.

(3) When the confidence level of uncertain demand variables decreases, the model will less consider the demand fluctuation of freight transportation, and the influence of the protection level on the revenue of container shipping companies will be reduced.

(4) The probability of occurrence when the uncertain demand follows the lognormal distribution is more concentrated compared with the normal distribution, the optimal robust slot allocation scheme is more stable under the heavy tail freight demand.

In addition to cargo demand, the shipping market faces many other uncertainties, such as shipping network, freight rate, and port location. Therefore, a future research direction is to incorporate more uncertainties into the model. Second, this study only considers a single shipping company and does not take liner alliance into account. However, according to the current trend of globalization, it is more realistic for shipping companies to make an alliance and mutual rent.

\section{REFERENCES}

[1] Yu, M.; Liao, J. (2021). Container slot allocation and dynamic pricing considering overbooking and market segmentation, IOP Conference Series: Earth and Environmental Science, Vol. 638, Paper 012026, 8 pages, doi:10.1088/1755-1315/638/1/012026

[2] Lu, H.-A.; Chu, C.-W.; Che, P.-Y. (2020). Seasonal slot allocation planning for a container liner shipping service, Journal of Marine Science and Technology, Vol. 18, No. 1, 84-92, doi: $10.51400 / 2709-6998.1868$

[3] Feng, C.-M.; Chang, C.-H. (2010). Optimal slot allocation with empty container reposition problem for Asia ocean carriers, International Journal of Shipping and Transport Logistics, Vol. 2, No. 1, 22-43, doi:10.1504/IJSTL.2010.029895

[4] Zurheide, S.; Fischer, K. (2015). Revenue management methods for the liner shipping industry, Flexible Services and Manufacturing Journal, Vol. 27, No. 2, 200-223, doi:10.1007/s10696-0149192-0

[5] Wang, Y.; Meng, Q.; Du, Y. (2015). Liner container seasonal shipping revenue management, Transportation Research Part B: Methodological, Vol. 82, 141-161, doi: $\underline{10.1016 /}$ J.TRB.2015.10.003

[6] Zhao, H.; Meng, Q.; Wang, Y. (2020). Probability estimation model for the cancellation of container slot booking in long-haul transports of intercontinental liner shipping services, Transportation Research Part C: Emerging Technologies, Vol. 119, Paper 102731, 19 pages, doi:10.1016/j.trc.2020.102731

[7] Wang, Y.; Meng, Q. (2019). Integrated method for forecasting container slot booking in intercontinental liner shipping service, Flexible Services and Manufacturing Journal, Vol. 31, No. 3, 653-674, doi:10.1007/s10696-018-9324-Z

[8] Wang, T.; Tian, X.; Wang, Y. (2020). Container slot allocation and dynamic pricing of timesensitive cargoes considering port congestion and uncertain demand, Transportation Research 
Ma, Lin, Ma: Data-Driven Robust Model for Container Slot Allocation with Uncertain Demand

Part E: Logistics and Transportation Review, Vol. 144, Paper 102149, 16 pages, doi:10.1016/j.tre.2020.102149

[9] Huang, Z. (2020). Slot allocation in container liner revenue management, International Core Journal of Engineering, Vol. 6, No. 10, 32-43, doi:10.6919/ICJE.202010_6(10).0006

[10] Zurheide, S.; Fischer, K. (2011). A revenue management slot allocation model with prioritization for the liner shipping industry, Hu, B.; Morasch, K.; Pickl, S.; Siegle, M. (Eds.), Operations Research Proceedings 2010, Springer, Berlin, 143-148, doi:10.1007/978-3-642-20009-0 23

[11] Hu, J.; Huang, Y.; Zhao, X. (2019). Research on the slot allocation of container liner under Ecommerce environment, Computers \& Industrial Engineering, Vol. 129, 556-562, doi:10.1016/ j.cie.2019.01.031

[12] Fu, Y.; Song, L.; Lai, K. K.; Liang, L. (2016). Slot allocation with minimum quantity commitment in container liner revenue management: A robust optimization approach, The International Journal of Logistics Management, Vol. 27, No. 3, 650-667, doi:10.1108/IJLM-06-2013-0075

[13] Ting, S.-C.; Tzeng, G.-H. (2016). Bi-criteria approach to containership slot allocation in liner shipping, Maritime Economics \& Logistics, Vol. 18, No. 2, 141-157, doi:10.1057/mel.2015.12

[14] Wang, T.; Xing, Z.; Hu, H.; Qu, X. (2019). Overbooking and delivery-delay-allowed strategies for container slot allocation, Transportation Research Part E: Logistics and Transportation Review, Vol. 122, 433-447, doi:10.1016/j.tre.2018.12.019

[15] Zhao, H.; Meng, Q.; Wang, Y. (2021). Robust container slot allocation with uncertain demand for liner shipping services, Flexible Services and Manufacturing Journal, 29 pages, Online first article, doi:10.1007/s10696-021-09420-z

[16] Soyster, A. L. (1973). Technical note-convex programming with set-inclusive constraints and applications to inexact linear programming, Operations Research, Vol. 21, No. 5, 1154-1157, doi:10.1287/opre.21.5.1154

[17] Mulvey, J. M.; Vanderbei, R. J.; Zenios, S. A. (1995). Robust optimization of large-scale systems, Operations Research, Vol. 43, No. 2, 264-281, doi:10.1287/opre.43.2.264

[18] Ben-Tal, A.; Nemirovski, A. (1999). Robust solutions of uncertain linear programs, Operations Research Letters, Vol. 25, No. 1, 1-13, doi:10.1016/S0167-6377(99)00016-4

[19] Bertsimas, D.; Sim, M. (2004). The price of robustness, Operations Research, Vol. 52, No. 1, 3553, doi:10.1287/opre.1030.0065

[20] Li, J. X.; Wen, X. N. (2020). Construction and simulation of multi-objective rescheduling model based on PSO, International Journal of Simulation Modelling, Vol. 19, No. 2, 323-333, doi:10.2507/IJSIMM19-2-CO8

[21] Yang, M.; Liu, Y.; Yang, G. (2020). Robust optimization for a multiple-priority emergency evacuation problem under demand uncertainty, Journal of Data, Information and Management, Vol. 2, No. 4, 185-199, doi:10.1007/s42488-019-00018-7

[22] Wang, Z.; Qi, M. (2020). Robust service network design under demand uncertainty, Transportation Science, Vol. 54, No. 3, 676-689, doi:10.1287/trsc.2019.0935

[23] Kim, J.; Chung, B. D.; Kang, Y.; Jeong, B. (2018). Robust optimization model for closed-loop supply chain planning under reverse logistics flow and demand uncertainty, Journal of Cleaner Production, Vol. 196, 1314-1328, doi:10.1016/j.jclepro.2018.06.157

[24] Liu, Y.; Lei, H.; Zhang, D.; Wu, Z. (2018). Robust optimization for relief logistics planning under uncertainties in demand and transportation time, Applied Mathematical Modelling, Vol. 55, 262280, doi:10.1016/j.apm.2017.10.041

[25] Ning, C.; You, F. (2018). Data-driven decision making under uncertainty integrating robust optimization with principal component analysis and kernel smoothing methods, Computers \& Chemical Engineering, Vol. 112, 190-210, doi:10.1016/j.compchemeng.2018.02.007

[26] Zhang, Y.; Feng, Y.; Rong, G. (2016). Data-driven chance constrained and robust optimization under matrix uncertainty, Industrial \& Engineering Chemistry Research, Vol. 55, No. 21, 61456160, doi:10.1021/acs.iecr.5b04973

[27] Zhang, Y.; Feng, Y.; Rong, G. (2017). New robust optimization approach induced by flexible uncertainty set: Optimization under continuous uncertainty, Industrial \& Engineering Chemistry Research, Vol. 56, No. 1, 270-287, doi:10.1021/acs.iecr.6b02989 